\title{
Optic sensors of high refractive-index responsivity and low thermal cross sensitivity that use fiber Bragg gratings of $>\mathbf{8 0}^{\circ}$ tilted structures
}

\author{
Kaiming Zhou, Lin Zhang, Xianfeng Chen, and Ian Bennion \\ Photonic Research Group, Aston University, Birmingham, B4 7ET, UK
}

Received January 19, 2006; revised January 26, 2006; accepted January 31, 2006; posted February 2, 2006 (Doc. ID 65494)

For the first time to the authors' knowledge, fiber Bragg gratings (FBGs) with $>80^{\circ}$ tilted structures have been fabricated and characterized. Their performance in sensing temperature, strain, and the surrounding medium's refractive index was investigated. In comparison with normal FBGs and long-period gratings (LPGs), $>80^{\circ}$ tilted FBGs exhibit significantly higher refractive-index responsivity and lower thermal cross sensitivity. When the grating sensor was used to detect changes in refractive index, a responsivity as high as $340 \mathrm{~nm} /$ refractive-index unit near an index of 1.33 was demonstrated, which is three times higher than that of conventional LPGs. (C) 2006 Optical Society of America

OCIS codes: $050.2770,060.2310$.

Optical fiber sensors based on fiber gratings, including Bragg and long-period structures, have been extensively investigated in the past decade. The sensing principle is based on the influence on the properties of mode coupling of variations in environmental parameters such as temperature, strain, and the surrounding medium's reflective index (SRI). However, another type of fiber grating, the tilted fiber Bragg grating (TFBG), is much less often used for sensing. TFBGs of small tilted structures can couple the light from core to backward-propagating corecladding-radiation modes. This property has been used in the design of in-fiber spectrometers ${ }^{1,2}$ and for wavelength-division multiplexing channel monitoring ${ }^{3}$ and gain flattening of erbium-doped fiber amplifiers. ${ }^{4}$ Because of their exceptional polarization sensitivity, TFBGs have also been used as in-line polarimeters, ${ }^{5,6}$ polarization-dependent loss equalizers, ${ }^{7}$ and polarizers. ${ }^{8}$ For these applications the grating structures were blazed at relatively large angles but $45^{\circ}$ at most. To date, we are aware of no report of fabrication of TFBGs with $>45^{\circ}$ tilted structures or their mode-coupling properties and applications. In this Letter we report the fabrication of TFBGs with tilted angles up to $81^{\circ}$ and our investigation of their forward mode-coupling property and responsivities to strain, temperature, and the SRI.

Light coupling in a TFBG can be well understood by analysis of the phase-matching condition as shown in Fig. 1. Coupling will be the strongest if the phase-matching condition, $\mathbf{K}_{R}=\mathbf{K}_{\text {core }}+\mathbf{K}_{G}$, is satisfied, where $\mathbf{K}_{R}, \mathbf{K}_{\text {core }}$, and $\mathbf{K}_{G}$ are wave vectors of the radiated light, core mode, and grating, respectively. In general, we may neglect the difference between the amplitudes of $\mathbf{K}_{R}$ and $\mathbf{K}_{\text {core }}$ because the refractive indices of core and cladding are close. The direction of the coupled light $\left(\mathbf{K}_{R}\right)$ depends on the tilted angle of the TFBG. As shown in Fig. 1(a), when the grating's tilted angle $\delta$ is $<45^{\circ}$, the core mode light is coupled to the backward-propagating direction; i.e., radiation angle $\theta$ is an obtuse angle $\left(>90^{\circ}\right)$. When the grating structure is tilted at $\delta>45^{\circ}$, radiation angle $\theta$ is an acute angle $\left(<90^{\circ}\right)$, indicating that the core light can be coupled to the forward-propagating direction. By taking the total internal reflection effect into consideration, we note that a tilt angle range exists for TFBGs in which the light will not be confined and propagate in the fiber but will be coupled out from the side of the grating. As shown in Fig. 1(c), we have calculated this range to be $23.1^{\circ}-66.9^{\circ}$ in air $(n \sim 1)$ and $11.5^{\circ}-78.5^{\circ}$ in a water-based solution $(n \sim 1.33)$. Below and beyond this range, the light will be coupled to backward- and forward-propagating cladding modes, respectively, and confined in the fiber. Conventionally, the forward cladding mode-coupling regime exists only in the long-period grating (LPG) structures, resulting in broad (typically $10-20 \mathrm{~nm}$ ) transmission resonances that are ultrasensitive to external conditions. Although the sensitivity of longperiod gratings (LPGs) has been utilized for implementation of optical sensors, their extreme cross sensitivity to temperature and banding limits their performance in real applications. It would be more interesting to compare the sensitivities of forwardcoupling TFBGs and LPGs and to identify the advantages of each. Thus we fabricated TFBGs with tilted angles of $>80^{\circ}$ and characterized their spectral responses to strain, temperature, and, especially, to refractive indices of water-based solutions.

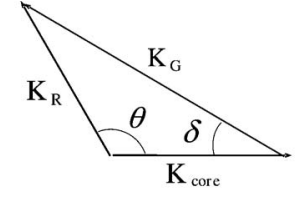

(a)

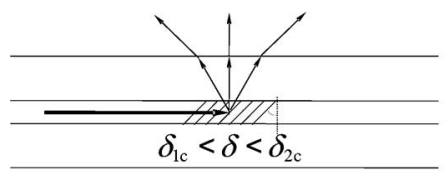

(c)

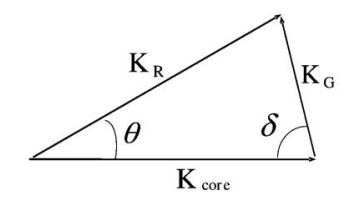

(b)

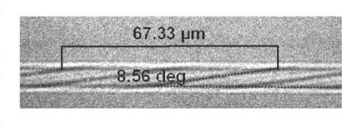

(d)
Fig. 1. Phase-matching conditions for (a) small tilted structures and backward-propagating coupling and (b) large tilted structures and forward-propagating coupling. (c) When the tilted angle satisfies $\delta_{1 c}<\delta<\delta_{2 c}$, radiation mode coupling is facilitated. (d) Image of tilted fringes of an $81^{\circ}$ TFBG. 


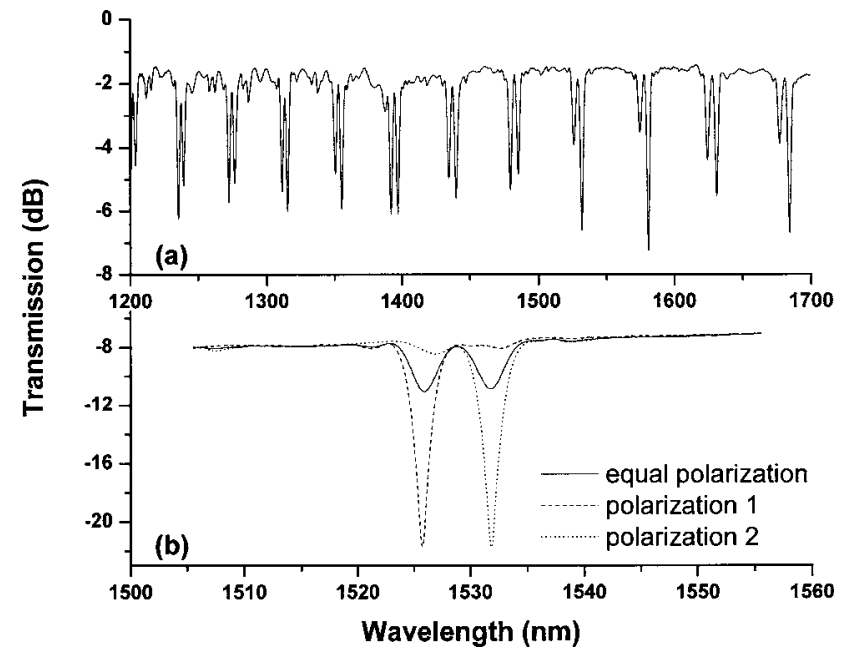

Fig. 2. (a) Series of dual-peak resonances from 1200 to $1700 \mathrm{~nm}$ with separation of $\sim 7 \mathrm{~nm}$ appear in the transmission spectrum of an $81^{\circ}$ TFBG. (b) The dual-peak feature is caused by the polarization state of the input light.

The TFBGs with the $>80^{\circ}$ tilted structure were UV inscribed in $\mathrm{H}_{2}$-loaded standard telecom fiber by use of a frequency-doubled $\mathrm{Ar}^{+}$laser and the scanning mask technique. A custom-designed mask of a period of $6.6 \mu \mathrm{m}$ was specially purchased to ensure that grating responses were positioned in our broadband light source from 1200 to $1700 \mathrm{~nm}$. In the UV inscription, the mask was tilted at $78^{\circ}$ to induce tilted fringes at $81.3^{\circ}$ in the fiber. ${ }^{7}$ Several such gratings with large tilts were fabricated, and their fringe structures were examined under a microscope of high magnification. Figure 1(d) shows an image of the tilted fringes at $\sim 81.3^{\circ}\left(90^{\circ}-8.67^{\circ}\right)$. The spectral responses of these TFBGs were then measured by use of the broadband light source and an optical spectrum analyzer. Figure 2 depicts the transmission spectrum of an $81^{\circ}$ TFBG, showing a set of dual-peak resonances distributed from 1200 to $1700 \mathrm{~nm}$ with a nearly even separation between adjacent resonances. In a close examination we can see that the spacings between the adjacent and paired modes are modeorder dependent. They are $\sim 60$ and $7 \mathrm{~nm}$ for the highest- and $\sim 40$ and $4 \mathrm{~nm}$ for the lowest-order modes, respectively, in the measuring wavelength range. In comparison with conventional LPGs, which have typical periods of several hundred micrometers, these resonances are much narrower, giving an advantage for measurement of high resolution. It can be seen from the spectrum that the strengths of the dual peaks are $\sim 3 \mathrm{~dB}$, which suggests that the light may be coupled into paired modes of two orthogonal polarization states. We verified that this was so by using a polarizer and a polarization controller in the measurement system to select the polarization state. We zoomed one of the paired peaks to $\sim 1525 \mathrm{~nm}$ and measured the polarization dependence. As shown in Fig. 2(b), with random polarization we see two $3-\mathrm{dB}$ peaks, and, when the light is switched to either polarization, one of the peaks disappears while the other grows into a strong peak of $14 \mathrm{~dB}$ attenuation.
The spectral separation induced by the polarization effect between the paired peaks is $\sim 6 \mathrm{~nm}$.

To evaluate the potential of these gratings for applications in sensing, we characterized them in terms of temperature, strain, and SRI. One of the TFBGs was heated from $0^{\circ} \mathrm{C}$ to $70^{\circ} \mathrm{C}$ in $5^{\circ} \mathrm{C}$ increments in an environmental chamber, and its transmission spectrum was monitored in situ. Figure 3(a) plots the wavelength shifts versus temperature for the two paired resonances of the highest-order (close to $1700 \mathrm{~nm}$ ) and the lowest-order (close to $1200 \mathrm{~nm}$ ) modes in the measurement range. The four measured peaks all exhibit a linear thermal response characteristic, showing temperature sensitivities of 3.3 , and $3.8 \mathrm{pm} /{ }^{\circ} \mathrm{C}$ and $7.5,7.8 \mathrm{pm} /{ }^{\circ} \mathrm{C}$, respectively, for the two paired modes. The former values are only half of those of the latter, indicating that the lower-order modes have lower thermal sensitivity. By comparison, we see that the temperature sensitivity values of the low-order modes of this $81^{\circ}$ tilted TFBG are 2.5 times lower than those (typically $10 \mathrm{pm} /{ }^{\circ} \mathrm{C}$ ) of FBGs with normal or small tilted structures. This suggests that the largely tilted TFBGs have intrinsically small thermal cross sensitivity, which awards a significant advantage to such grating devices, especially for sensing applications for which the temperature effect has to be eliminated.

We examined the refractive-index sensitivity of the forward-propagating cladding modes of $81^{\circ}$ tilted TFBGs, which is of a particular interest for implementing an optical biosensor. We measured SRIinduced wavelength changes by using a set of indexmatching gels for the lowest- and highest-order paired modes. We noted from the experiment that, as soon as the grating was submerged in the index gel, the paired peaks evolved into one strong peak and became polarization independent. The results are plotted in Fig. 3(b) and show clearly that, when the SRI changes from 1.3 to 1.4, the wavelength shift reaches almost $15 \mathrm{~nm}$ for the peak close to $1200 \mathrm{~nm}$ but $30 \mathrm{~nm}$ for the peak near $1700 \mathrm{~nm}$. This indicates that

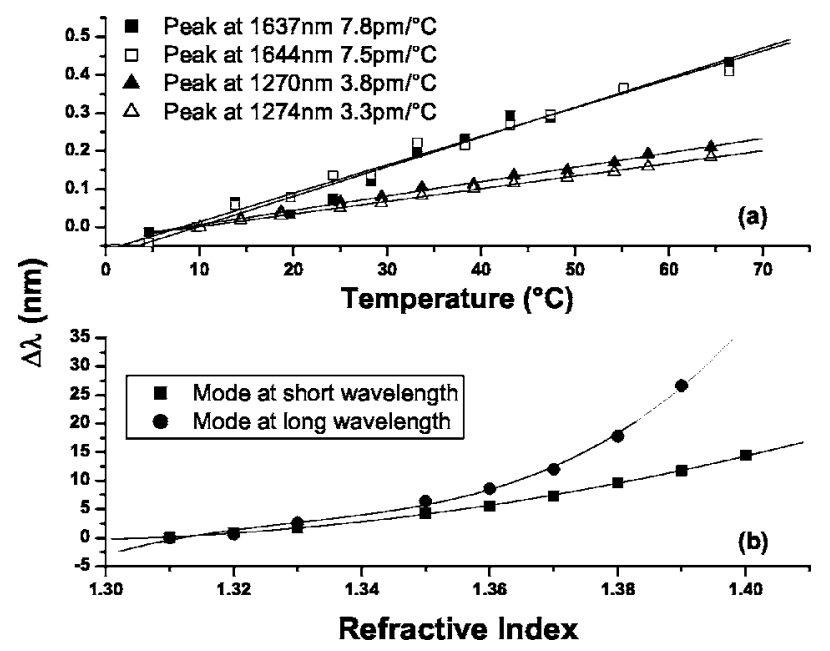

Fig. 3. (a) Wavelength shift versus temperature of the $81^{\circ}$ TFBG, showing a linear temperature response with a significantly lower thermal coefficient. (b) SRI-induced wavelength shifts in the index range from 1.3 to 1.4 for peaks at the shorter- and longer-wavelength sides, respectively. 
the SRI sensitivity of the excessively tilted TFBGs is also mode-order dependent: The lower-order mode has lower SRI sensitivity, and the sensitivity increases more dramatically for the high-order mode with increasing SRI. We estimate that the average index sensitivity of the peak at the longerwavelength side is $\sim 340 \mathrm{~nm} /$ refractive-index unit near 1.33 , which is six times higher than that of conventional LPGs, such as reported in Refs. 9 and 10. With a standard optical interrogation system of $0.1 \mathrm{~nm}$ resolution, this TFBG can be used to detect changes as small as $3 \times 10^{-4}$ in the SRI. Although we did not carry out the measurement further to $n$ $>1.4$, from the trends we can see that the resolution and sensitivity should be even higher in this region.

We also carried out an experiment to examine the strain responses of this $>80^{\circ}$ TFBG. The grating fiber was mounted on two metal block holders, one of which was fitted with a micrometer driver. We applied a strain of $0-2000 \mu \epsilon$ to the grating by stretching the fiber, and the wavelength shifts for all the peaks were recorded. Figure 4(a) plots the wavelength shift versus strain for one of the highest-order dual peaks, near $1680 \mathrm{~nm}$. The plot exhibits a linear relation, giving a strain coefficient of $1.8 \mathrm{pm} / \mu \epsilon$, which is slightly higher in value than that $(1.0 \mathrm{pm} / \mu \epsilon$ at $\sim 1550 \mathrm{~nm})$ of typical fiber Bragg gratings (FBGs) of normal structures. However, the coefficient is negative; i.e., the resonant peak moves to the shorter-wavelength side when the strain is increasing. This is contrary to the behavior of normal FBGs but in agreement with LPGs of relatively small periods. ${ }^{9}$ Figure $4(\mathrm{~b})$ plots the strain coefficient versus the peak number for eleven paired peaks in the monitoring range. We see that the peaks at the longer-wavelength side have larger values than those at the shorter-wavelength side, and one set of the polarized modes has higher strain coefficients than the other set.

From the results presented above, we can see that the $>45^{\circ}$ tilted TFBGs exhibit markedly different temperature, strain, and SRI sensitivity characteristics from the fiber Bragg gratings of normal and $<45^{\circ}$ tilted structures and conventional LPGs. However,

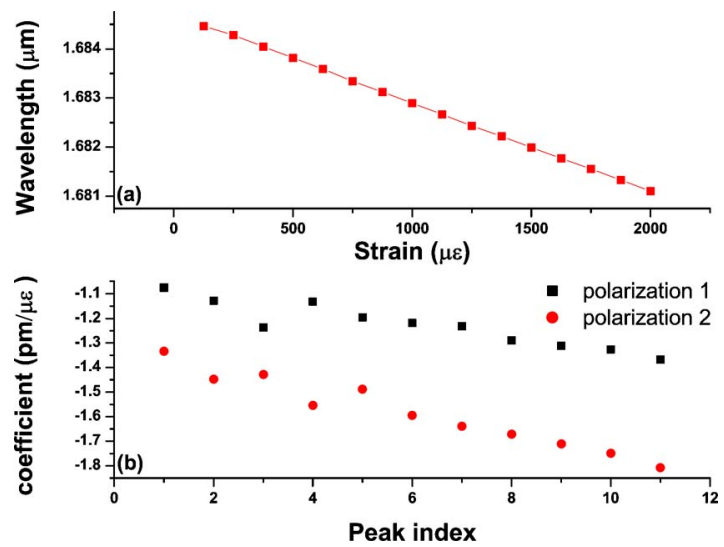

Fig. 4. (Color online) Strain responses of the $81^{\circ}$ TFBG: (a) linear and negative strain response for a peak at the shorter-wavelength side and (b) the wavelength shift caused by strain depends on mode order and polarization. they have shown similar responses, such as negative strain coefficients and high SRI sensitivity, to the LPGs of small periods $(<100 \mu \mathrm{m})$. This is not surprising, as the TFBGs are forward-mode-coupling devices. Given a phase mask period of $6.6 \mu \mathrm{m}$ and fabrication tilt angle $78^{\circ}$, we can calculate the grating period along the fiber axis as $\sim 32 \mu \mathrm{m}$. Thus these forward-coupling TFBGs in fact can be regarded as long-period gratings with much smaller period sizes that are capable of coupling light from the core to the high-order cladding modes with much closer mode separations, as shown in Fig. 2(a). However, the excessive-tilting structure contributes some unique effects e.g., a pronounced polarization effect, to the grating response. The largely tilted fringes obviously induce significant birefringence in the fiber, resulting in dual-peak resonances that correspond to the two orthogonal polarization states, as has been experimentally verified [Fig. 2(b)].

In conclusion, FBGs with $>80^{\circ}$ tilted structures have been fabricated and characterized. The optical responses of such gratings show forward-propagating operation and strong polarization dependence. Their responses to temperature, strain, and SRI have been evaluated, demonstrating a significantly lower thermal and higher strain and SRI sensitivities than normal or more slightly tilted FBGs and conventional LPGs. The intrinsically low thermal cross sensitivity and narrow spectral responses of such TFBGs are particularly attractive as optical sensors, which require low thermal cross sensitivity and high resolution measurement. In particular, their remarkably enhanced SRI sensitivity near 1.33 will be of interest for implementation of optical biosensors and applications.

\section{K. Zhou's e-mail address is k.zhou@osa.org.}

\section{References}

1. J. L. Wagener T. A. Strasser, J. R. Pedrazzani, J. Demarco, and D. J. Digivanni, in 23rd European Conference on Optical Communication, Conf. Publ 448, (Institute of Electrical Engineers, 1997), pp. 65-68.

2. K. S. Feder, P. S. Westbrook, J. Ging, P. I. Reyes, and G. E. Carver, IEEE Photon. Technol. Lett. 15, 933 (2003).

3. R. S. Westbrook, K. S. Feder, P. I. Reyes, P. Steinvurzel, B. J. Eggleton, R. G. Ernst, L. A. Reith, and D. M. Gill, Optical Fiber Communication Conference (OFC) (Optical Society of America, 2002), pp. 680-682, paper ThGG49.

4. R. Kashyap, R. Wyatt, and R. J. Campbell, Electron. Lett. 29, 154 (1993).

5. P. S. Westbrook, T. A. Strasser, and T. Erdogan, IEEE Photon. Technol. Lett. 12, 1352 (2000).

6. J. Peupelmann, E. Krause, A. Bandemer, and C. Schäffer, Electron. Lett. 38, 1248 (2002).

7. S. J. Mihailov, R. B. Walker, T. J. Stocki, and D. C. Johnson, Electron. Lett. 37, 284 (2001).

8. K. Zhou, A. G. Simpson, X. Chen, L. Zhang, and I. Bennion, Opt. Lett. 30, 1285 (2005).

9. X. Shu, B. A. L. Gwandu, Y. Liu, L. Zhang, and I. Bennion, Opt. Lett. 26, 774 (2001).

10. B. H. Lee, Y. Liu, S. B. Lee, S. S. Choi, and J. N. Jang, Opt. Lett. 22, 1769 (1997). 